\title{
Abnormality of the Foramen Spinosum due to a Variation in the Trajectory of the Middle Meningeal Artery: A Case Report in Human
}

\author{
Joel Henrique Ellwanger ${ }^{1}$ Deivis de Campos ${ }^{1,2}$ \\ ${ }^{1}$ Department of Biology and Pharmacy, Laboratory of Histology and \\ Pathology, University of Santa Cruz do Sul - UNISC, Santa Cruz do Sul, \\ RS, Brazil \\ 2 Institute of Basic Health Sciences, Post Graduate Program in \\ Neurosciences, Federal University of Rio Grande do Sul - UFRGS, RS, Brazil
}

\begin{abstract}
Address for correspondence Deivis de Campos, PhD, Departamento de Biologia e Farmácia, Laboratório de Histologia e Patologia, Universidade de Santa Cruz do Sul, Av. Independência, 2293, Santa Cruz do Sul, RS, Brazil (e-mail: dcampos@unisc.br).
\end{abstract}

J Neurol Surg Rep 2013;74:73-76.

\section{Introduction}

The majority of vascular anomalies of the head and neck remain unrecognized throughout the lifetime of a person. As a result of atherosclerotic vascular disease, senile changes, or some other pathologic process, anomalies that were asymptomatic may become symptomatic and of clinical importance, especially if the pathologic process requires angiologic examination or surgical treatment. ${ }^{1}$

Among the numerous anatomical variations of the head and neck that are of clinical interest, one deserves particular attention. The variations in the origin of the middle meningeal artery (MMA) are of clinical importance when dealing with fractures of the base of the skull, epidural hematomas, and surgery of the nerve of the pterygoid canal or the vidian nerve, as well as during endovascular and surgical interventions of the internal maxillary artery. ${ }^{2}$

received

December 26, 2012

accepted

March 25, 2013

published online

May 23, 2013
This artery, or its branches, and other large intracranial extracerebral vessels have been implicated in the pathophysiology of migraine by theories suggesting neurogenic inflammation or cranial vasodilatation, or both, as explanations for the pain of migraine. ${ }^{3}$ Moreover, some studies ${ }^{4,5}$ showed that detailed knowledge of the morphological organization of the MMA can assist in procedures like bypass.

The MMA is the largest and most important of the arteries supplying the meninges. It is usually a branch of the first part of the maxillary artery. However, anomalous courses, anastomoses, and origins of the MMA have often been described in the literature. ${ }^{6,7}$

In this study, we report an additional case of an abnormality of the foramen spinosum due to a variation in the trajectory of the MMA and discuss the anatomy and possible embryological explanations of this unusual vascular configuration. Moreover, the actual literature on the incidence of the
License terms Stuttgart · New York

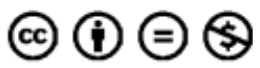


variations of the origin of the MMA and their embryological basis is reviewed.

\section{Case Report}

In a Caucasian cadaver skull of an approximately 35-year-old individual belonging to the didactical collection of the Laboratory of Human Anatomy at the University of Santa Cruz do Sul, Brazil, it was noted that the right foramen spinosum has an abnormal shape. The shape is like a channel $5.37 \mathrm{~mm}$ in length and $2.36 \mathrm{~mm}$ in diameter. In an unusual way, this channel has direct access to the foramen ovale. This abnormality was seen only on the right side and there was no sign of deformation in other skull bones. The diameter of the left foramen spinosum is $2.23 \mathrm{~mm}$. It is interesting to note that the diameters of the foramens ovale are significantly different. The foramen ovale on the right side has a larger diameter than the foramen ovale on the left side (-Fig. 1). The measurements were taken using a digital pachymeter from Digimess (São Paulo, SP, Brazil).
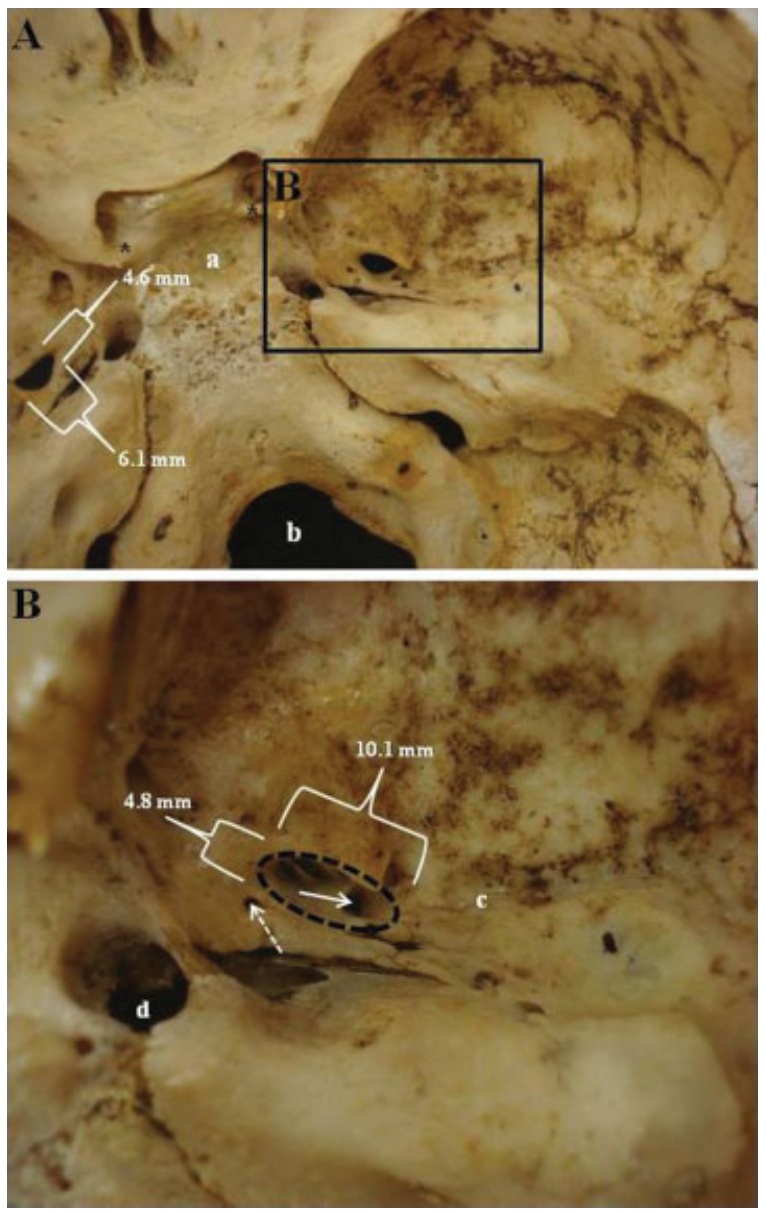

Fig. 1 Internal view of the skull (A) highlighting (B) the region of the foramen spinosum ( $B$, solid arrow) in relation to the foramen ovale ( $B$, dotted ellipse). Note the presence of calcification of the clinoid processes above ( $A$, asterisks), as well as the existence of an unusual foramen ( $B$, dotted arrow) near the foramen ovale. (a) Hypophyseal fossa. (b) Foramen magnum. (c) Groove of the middle meningeal artery. (d) Foramen lacerum.
Our observations also verified the presence of calcification of the clinoid processes above, as well as the presence of an unusual foramen near the foramen ovale (-Fig. 1). The possible explanations for these additional abnormalities in the skull will not be discussed in this study because they are not part of the focus of this case report.

\section{Discussion}

\section{Usual Anatomical Aspects of the MMA}

The MMA normally enters the cranium through the foramen spinosum of the sphenoid bone after originating from the internal maxillary branch of the external carotid artery. The main trunk runs anteriorly in a groove on the greater wing of the sphenoid bone before dividing into two major branches, anterior and posterior. In addition, several smaller branches originate near the foramen spinosum. The ganglionic branches, usually tiny, supply the trigeminal ganglion, roots, and adjacent dura. A petrosal branch to the greater petrosal nerve runs through the facial hiatus and anastomoses with the stylomastoid branch of the external carotid artery. The superior tympanic branch supplies the tensor tympani coursing through its canal. ${ }^{7}$

\section{Embryological Basis of the MMA}

Some reports about the anatomic variations of the MMA regarding the origin, position, and intracranial branches ${ }^{8,9}$ and several anomalies of this artery ${ }^{6,10-12}$ have been reported for more than three decades; before that, embryological studies had been conducted to improve knowledge of possible anatomic variations of this important arterial segment. ${ }^{13}$

Liu and Rhoton ${ }^{14}$ described that the complex embryology underlying the development of the MMA may give rise to several anomalous origins, courses, and anastomoses involving this vessel. The authors reported that variants included origin of the MMA from the ophthalmic or internal carotid artery, ophthalmic artery origin from the MMA, and meningolacrimal anastomosis, all of which have important clinical implications for endovascular therapy of skull base lesions.

At the fourth to fifth weeks of gestation, the MMA begins as a dorsal branch of the stapedial artery, which arises from the hyoid artery derived from the second branchial arch. After passing through the ring of stapes, it divides into two main branches: the maxillofacial, which leaves the cranial cavity through the foramen spinosum, and the supraorbital branch that supplies the orbit and the intracranial segment of the MMA anteriorly. At the seventh to eighth weeks of gestation, the stapedial artery involutes and the remnants become the tympanic branches of the MMA, which retain communication with the supraorbital and maxillofacial divisions. Thus, the distal portion of the internal maxillary artery forms and communication with the intracranial components of the stapedial system is established. The branches of the normal adult MMA includes a supraorbital branch to the orbit, as well as smaller branches to the trigeminal ganglion, the petrosal branch, and the superior tympanic artery. ${ }^{2}$

According to studies performed by Altmann ${ }^{15}$ and Manjuanth, ${ }^{6}$ the MMA may arise as a branch of the persistent 
stapedial artery, but just rarely. These authors reported that the persistent stapedial artery, usually a branch of the petrous portion of the internal carotid artery, enters the tympanic cavity through its floor and traverses the obturator foramen of the stapes. It is enclosed in a bony canal for a short distance and passes through the facial canal to emerge into the middle cranial fossa under the dura to give off the MMA.

\section{Anomalous Origin of the MMA}

In our study, beyond the description of an unusual shape of the foramen spinosum, we found that the right foramen ovale is significantly greater than the left. We believe that this difference might be explained by the fact that the MMA would be entering the skull through the foramen ovale, since the channel reported in our study allows direct access to the foramen ovale and thus, presumably, the MMA would induce the enlargement of the foramen ovale. Chandler and Derezinski ${ }^{16}$ described that in the absence of foramen spinosum, the MMA may enter the middle cranial fossa through the foramen ovale.

Various sources of anomalous origin of the MMA have been identified in the medical literature. Bruner and Sherkat ${ }^{17}$ reported that the earliest cases of MMA arising from the ophthalmic artery were described by Curnow in 1874 and by Zukerkandl in 1876. The authors cited that, in some cases, this anomaly is associated with the absence of any external carotid artery contribution to the MMA and agenesis of the foramen spinosum. In the 1980s, a study performed by Dilenge and Ascherl ${ }^{18}$ reported 17 cases of MMA arising from the ophthalmic artery among 3.500 cerebral angiograms examined.

Shah and Hurst ${ }^{7}$ described an MMA originating from a large lateral pontine branch of the basilar artery. The origin of the MMA from the basilar artery or its branches is, however, extremely rare, with only five previous angiographic descriptions. According to the authors, the angiographic appearance of three cases of left MMA origin from the basilar artery were described in 1976. Subsequently, two additional angiographic demonstrations of this anomaly were reported, one of which was bilateral. The same researchers said that all of these cases shared features seen in the current case, with MMA origin from an enlarged lateral pontine branch, which in turn originated 5 to $10 \mathrm{~mm}$ proximal to the origin of the superior cerebellar artery and superior to the anterior inferior cerebellar artery.

With a postmortem dissection of a 7-month-old fetus, Altmann ${ }^{15}$ reported the very first case of MMA origin from basilar artery. Waga et al $^{19}$ published a single case of basilarMMA anastomosis in a 47-year-old man during angiography. Recently, Kuruvilla et al $^{2}$ described the first angiographically documented case of the anomalous origin of the MMA from the posterior inferior cerebellar artery. According to the authors, the posterior inferior cerebellar artery-MMA anastomosis can be obscured by arteriovenous malformations and vascularized tumors involving the dura of the posterior cranial fossa. From a neurosurgical and endovascular point of view, during the embolization of the MMA, knowledge of this type of anomalous connection would be useful for preventing inadvertent embolization of vital brain structures in the posterior fossa. These same authors described some sources of anomalous origins of the MMA: ascending pharyngeal artery, ophthalmic artery, extradural portion of the internal carotid siphon, lacrimal artery, and branch of the persistent stapedial artery.

\section{Current Neurosurgery and Clinical Relevance}

Epidural hematoma is a traumatic accumulation of blood between the inner table of the skull and the stripped-off dural membrane. The inciting event is often a direct blow to the head. In 85 to $95 \%$ of patients, this type of trauma results in an overlying fracture of the skull. ${ }^{17}$

Important information was reported in the study published by Zasler et $\mathrm{al}^{20}$ : blood vessels in close proximity to the fracture are the sources of the hemorrhage in the formation of an epidural hematoma. Because the underlying brain has usually been minimally injured, prognosis is excellent if treated aggressively. Outcome from surgical decompression and repair is related directly to a patient's preoperative neurological condition. Epidural hematoma occurs in 1 to $2 \%$ of all head trauma cases and in approximately $10 \%$ of patients who go into a traumatic coma. The temporoparietal region and the MMA are involved most commonly (66\%), although the anterior ethmoidal artery may be involved in frontal injuries, the transverse or sigmoid sinus in occipital injuries, and the superior sagittal sinus in traumas at the vertex. Bilateral epidural hematomas account for 2 to $10 \%$ of all acute epidural hematomas in adults but are exceedingly rare in children. Posterior fossa epidural hematomas represent $5 \%$ of all cases of epidural hematomas.

The MMA represents the source of bleeding in $85 \%$ of epidural hematomas. The endocranial arterial blood accumulation dislocates the cerebral structures that herniate and cause fatal brainstem compression. Therefore, the epidural hematoma is a life-threatening complication of skull fractures and needs to be surgically evacuated. In supratentorial hematomas, surgical treatment includes a bone flap made over the greater diameter of the clot, with the exposure of the foramen spinosum. After removing the blood clot, the MMA is ligated as close as possible to the foramen, to stop the arterial bleeding from its main trunk. In the absence of brain injuries, a dramatic recovery of neurological impairment is usually observed soon after the surgical procedure. The patient is frequently discharged within a week after surgery. At present, no functional impairments are known to be associated with the suppression of the middle meningeal vascularization. ${ }^{17}$

Most attention has been directed to anastomosis with the ophthalmic artery through the superior orbital fissure. This anastomosis can be so large that the lacrimal artery or the ophthalmic artery actually gives origin to the MMA. Thus, manipulations of the maxillary artery can cause dangerous irreparable complications including blindness if the ophthalmic artery originates from the MMA. In injuries of the MMA, one must be aware of the variations of its branches as well as of its anastomoses. This is very important for the fact that the ligature or clipping of the MMA for bleeding after injury will 
be totally or partially insufficient in many of the described case of mentioned variations. ${ }^{21}$

Lastly, Kresimir Lukic et al $^{1}$ described the presence of the aberrant MMA, and its branches may have serious implications in transantral surgery, radical maxillectomy, lateral approaches to the infratemporal and pterygopalatine fossae, and anterior and medial portions of the skull base (including lesions of the nasopharynx and oropharynx), as well as the dorsocaudal approach to the medial skull base.

\section{Conclusion}

More than a simple anatomical variation of the skull involving the MMA, our study provides essential information for the detailed knowledge of the anatomical variations of the origin of this important arterial segment. These variations are of clinical significance in fractures of the squamous and petrous parts of the temporal bones and in surgical interventions involving the nerve of the pterygoid canal and maxillary artery. Thus, this study shall be important for clinicians, surgeons, and academicians who handle this particular anatomical site. Moreover, this work aimed to provide a simple but multidisciplinary synthesis of the current knowledge concerning the morphogenesis, variation, and clinical significances of the MMA, and finally to help promoting future studies in this area.

\section{References}

1 Kresimir Lukic I, Gluncic V, Marusic A. Extracranial branches of the middle meningeal artery. Clin Anat 2001;14(4):292-294

2 Kuruvilla A, Aguwa AN, Lee AW, Xavier AR. Anomalous origin of the middle meningeal artery from the posterior inferior cerebellar artery. J Neuroimaging 2011;21(3):269-272

3 Hoskin KL, Zagami AS, Goadsby PJ. Stimulation of the middle meningeal artery leads to Fos expression in the trigeminocervical nucleus: a comparative study of monkey and cat. J Anat 1999;194 (Pt 4):579-588

4 Ustun ME, Buyukmumcu M, Ulku CH, Guney O, Salbacak A. Transzygomatic-subtemporal approach for middle meningeal-toP2 segment of the posterior cerebral artery bypass: an anatomical and technical study. Skull Base 2006;16(1):39-44
5 Ustün ME, Büyükmumcu M, Seker M, Karabulut AK, Uysal II, Ziylan T. Possibility of middle meningeal artery-to-petrous internal carotid artery bypass: an anatomic study. Skull Base 2004;14 (3):153-156

6 Manjunath KY. Anomalous origin of the middle meningeal artery A review. J Anat Soc India 2001;50:179-183

7 Shah QA, Hurst RW. Anomalous origin of the middle meningeal artery from the basilar artery: a case report. J Neuroimaging 2007;17(3):261-263

8 Lasjaunias P, Moret J, Manelfe C, Théron J, HassoT, Seeger J. Arterial anomalies at the base of the skull. Neuroradiology 1977;13 (5):267-272

9 Krmpotić-Nemanić J, Draf W, Helms J. Surgical anatomy of the head-neck region. Chirurgische Anatomie des Kopf-Hals-Bereiches. Berlin: Springer Verlag; 1985

10 Royle G, Motson R. An anomalous origin of the middle meningeal artery. J Neurol Neurosurg Psychiatry 1973;36(5):874-876

11 Seeger JF, Hemmer JF. Persistent basilar/middle meningeal artery anastomosis. Radiology 1976;118(2):367-370

12 Tanohata K, Maehara T, Noda M, Katoh H, Sugiyama S, Okazaki A. Anomalous origin of the posterior meningeal artery from the lateral medullary segment of the posterior inferior cerebellar artery. Neuroradiology 1987;29(1):89-92

13 Padget DH. The development of the cranial arteries in the human embryo. Contrib Embryol 1948;32:205-261

14 Liu Q Rhoton AL Jr. Middle meningeal origin of the ophthalmic artery. Neurosurgery 2001;49(2):401-406, discussion 406-407

15 Altmann F. Anomalies of the internal carotid artery and its branches; their embryological and comparative anatomical significance; report of a new case of persistent stapedial artery in man. Laryngoscope 1947;57(5):313-339

16 Chandler SB, Derezinski CF. The variations of the middle meningeal artery within the middle cranial fossa. Anat Rec 1935;62: 309-319

17 Bruner E, Sherkat $\mathrm{S}$. The middle meningeal artery: from clinics to fossils. Childs Nerv Syst 2008;24(11):1289-1298

18 Dilenge D, Ascherl GF Jr. Variation of the ophthalmic and middle meningeal arteries: relation to the embryonic stapedial artery. AJNR Am J Neuroradiol 1980;1:45-54

19 Waga S, Okada M, Yamamoto Y. Basilar-middle meningeal arterial anastomosis. . Case report. J Neurosurg 1978;49(3):450-452

20 Zasler ND, Katz DI, Zafonte RD. Brain injury medicine: principles and practice. New York: Demos Medical Publishing2006

21 Klisović D, Šikić E, Krmpotić-Nemanić J. Variations of the middle meningeal artery: significance for surgery and practice. Clin Anat 1993;6:289-294 